\title{
Grazing incidence X-ray diffraction and transmission electron microscopy studies on the oxide formation of molybdenum in water vapor environment
}

Ming Tang*, Andrew T. Nelson, Elizabeth S. Wood, Stuart A. Maloy

Materials Science and Technology Division, Los Alamos National Laboratory, Los Alamos, NM 87545, USA

Ying-Bing Jiang

TEM Laboratory, University of New Mexico, Albuquerque, NM 87131, USA

\begin{abstract}
To evaluate the feasibility of molybdenum as light water reactor nuclear fuel cladding, the oxidation behavior was tested in water vapor at elevated temperatures. In this study, we present experimental results on the oxidation and volatilization of molybdenum using various characterization techniques including grazing incidence $\mathrm{X}$-ray diffraction and transmission electron microscopy. After oxidation testing in water vapor at $600{ }^{\circ} \mathrm{C}$, experiment results show the formation of both $\mathrm{MoO}_{3}$ and $\mathrm{MoO}_{2}$. With the increasing temperature, $\mathrm{MoO}_{2}$ is the final oxidation product between 800 and $1200{ }^{\circ} \mathrm{C}$, and $\mathrm{MoO}_{3}$ is volatile at the temperatures.
\end{abstract}

Key words: molybdenum, oxidation, water vapor, GIXRD, TEM

* Corresponding author: Tel.: +1-505-665-1472; fax: +1-505-667-8021

Corresponding author e-mail address: mtang@lanl.gov 
Recent interest in the development of accident tolerant nuclear fuels requires alternative cladding materials to replace current zirconium alloys. One of many key material performance challenges for future accident tolerant core materials is its resistance to high temperature oxidation in steam .The excellent high temperature mechanical properties and thermal conductivity of molybdenum and its alloys provide attractive features as candidates for a next-generation cladding material. This cladding must not only be successful in service under long exposure to pressurized water at elevated temperature $\left(\sim 400^{\circ} \mathrm{C}\right)$ during normal operation, but also be resistant to water vapor and hydrogen at higher temperatures $\left(\sim 1000^{\circ} \mathrm{C}\right)$ under accident conditions [1,2]. Although previous studies [3-10] revealed a poor resistance to oxidation for molybdenum in air, less is known about the governing mechanisms and the kinetics of oxidation in steam related to the environment under loss of cooling accident (LOCA) conditions. The degree to which oxidation of molybdenum in water vapor compares to previous observations carried out in air and oxygen will help us evaluate the possibility of molybdenum as a candidate accident tolerant cladding material. In our study, molybdenum was characterized following steam oxidation testing under both normal operating conditions and representative LOCA conditions.

Oxidation of molybdenum is complex and involves several processes due to the fact that many oxides of molybdenum exist [4, 8-10]. Based on the Mo-O phase diagram [11], the principal stable oxide phases are $\mathrm{MoO}_{3}$, which melts at $800 \pm 5^{\circ} \mathrm{C}$, and $\mathrm{MoO}_{2}$, which melts at $\sim 2200{ }^{\circ} \mathrm{C}$. $\mathrm{MoO}_{3}$ adopts a layered structure with orthorhombic symmetry (space group: Pbnm, a $=0.3963$ $\mathrm{nm}, \mathrm{b}=1.396 \mathrm{~nm}$, and $\mathrm{c}=0.3969 \mathrm{~nm}$ ), which is composed of corner-sharing $\mathrm{MoO}_{6}$ octahedra; while $\mathrm{MoO}_{2}$ has a slightly distorted rutile structure (space group: $P 2_{1}, \mathrm{a}=0.5608, \mathrm{~b}=0.4842$, $\mathrm{c}=0.5517, \beta=119.75^{\circ}$ ), which is composed of edge-sharing $\mathrm{MoO}_{6}$ octahedra. Many intermediate oxides (e.g., $\mathrm{Mo}_{4} \mathrm{O}_{11}$ ) have been observed [11], but are not stable at elevated temperatures 
relevant to LWR operation. Molybdenum does not visibly react with oxygen or water at room temperature, and $\mathrm{MoO}_{3}$ is known to dominate oxidation of $\mathrm{Mo}$ in air. The trioxide is volatile and sublimates at high temperatures $\left(>600^{\circ} \mathrm{C}\right) . \mathrm{MoO}_{3}$ and $\mathrm{MoO}_{2}$ are nearly equally thermodynamically favorable to form across the temperature regime and there is a $\mathrm{MoO}_{3}-\mathrm{MoO}_{2}$ eutectic at $778{ }^{\circ} \mathrm{C}$ [9]. Therefore, more complexities are added to the oxidation process of molybdenum, especially which is truly depended on specific conditions including temperature, pressure, time, and medium (steam vs. air).

The autoclave testing and thermogravimetric testing on molybdenum in water vapor was reported and the oxide formation was discussed based on the measured weight change in a previous paper [12]. It revealed that the kinetics of oxidation and volatilization of molybdenum at elevated temperatures is severely delayed in water vapor-containing atmospheres compared to that observed in strongly oxidizing atmosphere. However, the previous work limited its focus to thermogravimetric analysis alone and did not investigate the phases present following oxidation. The focus of the present paper is on characterization of oxide formation of molybdenum in a water vapor environment up to $1200{ }^{\circ} \mathrm{C}$, especially by means of grazing incidence $\mathrm{X}$-ray diffraction (GIXRD) in combination with electron diffraction and high resolution transmission electron microscopy (HRTEM) techniques. The formation of $\mathrm{MoO}_{3}$ and $\mathrm{MoO}_{2}$ at $600{ }^{\circ} \mathrm{C}$ was observed under steam environment, then $\mathrm{MoO}_{2}$ is stable up to $1200{ }^{\circ} \mathrm{C}$ and $\mathrm{MoO}_{3}$ is volatilized as temperature increases from $600{ }^{\circ} \mathrm{C}$ to $800{ }^{\circ} \mathrm{C}$. Oxidation of molybdenum in air condition was also studied for a comparison.

Molybdenum sheet (Eagle Alloys, 99.9\%) was used in the current study for oxidation testing. The $0.25 \mathrm{~mm}$ molybdenum sheet was sectioned into rectangles nominally $5 \times 15 \mathrm{~mm}$ in size. The 
samples were then immersed in acetone and then cleaned using methanol and lint-free wipes. Samples tested under two atmospheres are discussed in this paper. First, oxidation studies were performed in synthetic air (20\% oxygen carried in argon). The synthetic air mixture used for this study was flowed at $200 \mathrm{~mL} / \mathrm{min}$ during the isothermal test periods. Second, $100 \%$ water vapor at atmospheric pressure using a high precision vapor generator (ASTEAM DV2MK, Bronkhorst High Tech Instruments, Ruurio, The Netherlands). Water vapor flowed at $5.26 \mathrm{~g} / \mathrm{h}$ through a heated transfer line reaching the sample chamber. The exposure times at each temperature were dictated by the loss rate of each test condition as described in previous publication [12].

The velocity of the oxidizing media can play an important role in the oxidation of some alloy systems. If the local partial pressure of the oxidizing medium (e.g. oxygen, water vapor) is the rate-limiting actor that governs kinetics, increased flow velocity has been shown to dictate response [13]. The test configuration used to oxidize Mo samples in the current study prevents a precise calculation of the flow velocity of the injected water vapor or other gas mixtures due to complex internal furnace geometry. Water vapor was injected into the furnace at a velocity of $0.48 \mathrm{~m} / \mathrm{s}$. However, the increase in diameter at the location in the furnace where the sample is exposed will result in an order of magnitude reduction in this value. Future investigations that focus specifically on the kinetics of oxidation will require an improved ability to identify the velocity of steam or other media, but as the focus of the present work is identification of the resulting oxide structures rather than specific kinetics, no additional evaluation of the local gas velocities has been performed.

GIXRD, scanning electron microscopy (SEM) and TEM were used to characterize the phase composition and structural evolution in the test samples. GIXRD measurements were performed using a Bruker AXS D8 advanced X-ray diffractometer at an X-ray incidence angle of $\alpha=0.5^{\circ}$. 
Under these conditions, $\mathrm{X}$-rays are scattered from the near surface of these samples within a depth usually less than $200 \mathrm{~nm}$ [14]. SEM and Energy-Dispersive X-ray Spectroscopy (EDS) measurements were performed with a FEI Inspect SEM. Test samples were prepared in crosssectional geometry for TEM examination using a focused-ion-beam (FIB) apparatus, based on the in situ lift out method. Microstructure evolution was examined using a FEI Tecnai F30 electron microscope operating at $300 \mathrm{kV}$.

Figure 1(a) shows the evolution of GIXRD patterns of molybdenum before and after oxidation tests in synthetic air at varying temperatures. Crystalline phase identification based on GIXRD patterns was aided by ICDD-PDF database [15]. The patterns obtained from pristine a Mo sample consist primarily of (110), (200), and (211) reflections representative of pure Mo phase with body-centered cubic (BCC) structure (JCPDF card 00-042-1120). At temperatures of 500 ${ }^{\circ} \mathrm{C}$ and $600{ }^{\circ} \mathrm{C}$ (isothermal exposure time $\sim 240$ minutes), oxidation of molybdenum to $\mathrm{MoO}_{3}$ (s) via the below reaction [4] (the HSC [16] calculated Gibbs energies of $-523.646 \mathrm{kj} / \mathrm{mol}$ at $600{ }^{\circ} \mathrm{C}$ ) was observed.

$2 \mathrm{Mo}(\mathrm{s})+3 \mathrm{O}_{2}(\mathrm{~g}) \rightarrow 2 \mathrm{MoO}_{3}(\mathrm{~s})$

With increasing temperature, $\mathrm{BCC}$ molybdenum reflections are greatly diminish. It suggests that oxidation of $\mathrm{Mo}$ to $\mathrm{MoO}_{3}$ is almost complete in GIXRD sampled area.

In Figure 1 (b), the evolution of GIXRD patterns of molybdenum before and after oxidation tests in $100 \%$ water vapor environment for temperatures at 600,800 , and $1200{ }^{\circ} \mathrm{C}$ (isothermal exposure times at $240,180,30$ minutes, respectively) is presented. New GIXRD reflections, other than those corresponding to the BCC phase of molybdenum, become visible in the GIXRD pattern obtained from the sample heated to $600{ }^{\circ} \mathrm{C}$ in $\mathrm{H}_{2} \mathrm{O}$. These new peaks can be indexed as 
corresponding to orthorhombic phase of $\mathrm{MoO}_{3}$ (JCPDF card 00-035-0609) and monoclinic phase of $\mathrm{MoO}_{2}$ (JCPDF card 01-076-1807). When the oxidation of molybdenum to $\mathrm{MoO}_{3}(\mathrm{~s}, \mathrm{~g}$ ) proceeds under water vapor, hydrogen is additionally produced (Equation 2[12], the HSC calculated Gibbs energies of $75.311 \mathrm{kj} / \mathrm{mol}$ for $600^{\circ} \mathrm{C}$ ).

$\mathrm{Mo}(\mathrm{s})+3 \mathrm{H}_{2} \mathrm{O}(\mathrm{g}) \rightarrow \mathrm{MoO}_{3}(\mathrm{~s}, \mathrm{~g})+3 \mathrm{H}_{2}(\mathrm{~g})$

Speiser and St. Pierre reported [9] that they observed a thin $\mathrm{MoO}_{2}$ layer, or an external $\mathrm{MoO}_{3}$ layer coexisting with a buried $\mathrm{MoO}_{2}$ layer on the surface of oxidized molybdenum. They interpreted the formation of $\mathrm{MoO}_{2}$ as the transformation from $\mathrm{MoO}_{3}$ to $\mathrm{MoO}_{2}$ via reaction 3:

$2 \mathrm{MoO}_{3}(\mathrm{~s})+\mathrm{Mo}(\mathrm{s}) \rightarrow 3 \mathrm{MoO}_{2}(\mathrm{~s})$

In another early study, Gulbransen et al. [4] revealed that $\mathrm{MoO}_{3}$ not only can be reduced to $\mathrm{MoO}_{2}$ at all temperatures by $\mathrm{Mo}$, but also can dissociate to $\mathrm{MoO}_{2}$ above its melting temperature $795^{\circ} \mathrm{C}($ Equation 4$)$.

$2 \mathrm{MoO}_{3}(\mathrm{~s}) \rightarrow 2 \mathrm{MoO}_{2}(\mathrm{~s})+\mathrm{O}_{2}(\mathrm{~g})$

When the temperature is raised to $800{ }^{\circ} \mathrm{C}$, almost all $\mathrm{MoO}_{3}(\mathrm{~s}, \mathrm{~g})$ is volatilized or transformed to $\mathrm{MoO}_{2}(\mathrm{~s})$ because there are no observable $\mathrm{MoO}_{3}$ peaks and only $\mathrm{MoO}_{2}$ reflections other than molybdenum peaks. At the highest temperature of $1200{ }^{\circ} \mathrm{C}$, GIXRD observation shows that $\mathrm{MoO}_{2}(\mathrm{~s})$ phase still exists, which is consistent with the well-known mechanism governing oxidation and volatilization processes of molybdenum, in which $\mathrm{MoO}_{2}$ is more stable, and nonvolatile oxide. Unlike the observation of samples in air, another important feature of GIXRD patterns of Mo in steam is that $\mathrm{BCC}$ molybdenum reflections are the dominant phase in the range at $600{ }^{\circ} \mathrm{C}$. It suggests that reduced oxidation of molybdenum occurs in water vapor than in 
synthetic air at the same temperature. This result agrees well with the weight change results for samples exposed to air and $\mathrm{H}_{2} \mathrm{O}$ in previous paper [12].

Figure 2(a) shows a $25-\mu \mathrm{m}$ thick layer with external crystal growth on molybdenum sample tested in synthetic air at a temperature of $600{ }^{\circ} \mathrm{C}$. EDS data (not shown here) indicates this crystal layer is composed of molybdenum oxides, in which the oxygen to molybdenum ratio is between 2 and 3. It is difficult to identify the exact stoichiometry of the oxide based on EDS analysis. SEM secondary electron image shows the nucleation and crystal growth of the regular shaped oxide grains.

Figure 2(b) shows that there is no observable oxide layer or crystal growth on molybdenum sample tested in water vapor at a temperature of $600{ }^{\circ} \mathrm{C}$. Similar observations are obtained from samples at $800^{\circ} \mathrm{C}$ and higher (data not shown here). It is in good agreement with our GIXRD observations, in which we understand the much less oxidation of Mo occurs in water vapor than in air at the same temperature.

Figure 3(a) shows a bright-field (BF) TEM micrograph from a Mo sample in water vapor at 600 ${ }^{\circ} \mathrm{C}$. There are some residual crystalline particles existing in the top of sample. Also shown in Fig. 3(a) are a series of SAED patterns obtained from top particles (labeled as $\mathrm{MoO}_{2}$ ), surface area (labeled as $\mathrm{MoO}_{3}$ ), and inside of sample (labeled as Mo), respectively. The inside SAED pattern corresponds to the cubic phase of molybdenum and is obtained along the beam direction of [113], while other two patterns obtained from particles and surface are complicated and possess two sets of diffraction spots. In SAED pattern of particles, the strong set of diffraction belongs to BCC Mo phase on the [113] zone axis, and the weak diffraction can be indexed as monoclinic $\mathrm{MoO}_{2}$ phase along the beam direction of [1-11]. In addition to the primary strong diffraction of 
Mo in SAED pattern of surface area, the weak set of diffraction coincides with the reciprocal lattice projected on the $\mathrm{MoO}_{3}$ [5-12] zone axis. Diffraction analysis reveals that there are $\mathrm{MoO}_{2}$ and $\mathrm{MoO}_{3}$ phases formed in top surface area.

Fig. 3(b) shows high resolution TEM images obtained from the surface of a Mo sample in water vapor at $600{ }^{\circ} \mathrm{C}$. This micrograph is obtained with the electron beam oriented along [113] with respect to BCC Mo phase. Also shown in Fig. 3(b) are magnified images obtained from three different crystalline domains in the micrograph (denoted as $\mathrm{Mo}, \mathrm{MoO}_{2}$, and $\mathrm{MoO}_{3}$ ). The interplanar spacings of $0.235,0.385,0.494 \mathrm{~nm}$ are measured from well-defined lattice fringes in these three magnified images labeled as $\mathrm{Mo}, \mathrm{MoO}_{3}$, and $\mathrm{MoO}_{2}$, respectively. By referring to the table of interplanar spacings, the measured three lattice planes match those of the (110) of BCC molybdenum crystal, the (110) of orthorhombic $\mathrm{MoO}_{3}$ phase, and (-101) plane of monoclinic $\mathrm{MoO}_{2}$ phase, which theoretically have d-spacings of $0.223 \mathrm{~nm}, 0.381 \mathrm{~nm}$, and $0.480 \mathrm{~nm}$, respectively. The evaluation of distances of the lattice fringes confirms the presence of $\mathrm{MoO}_{2}$ and $\mathrm{MoO}_{3}$ phases.

Both electron diffraction and HRTEM analysis confirm that there are $\mathrm{MoO}_{3}$ and $\mathrm{MoO}_{2}$ formed in the surface of Mo sample in water vapor at $600{ }^{\circ} \mathrm{C}$. This is consistent with GIXRD observation.

The BF TEM image (left), corresponding SAED pattern (top right), and HRTEM image (bottom right) of surface of Mo sample in water vapor at $800^{\circ} \mathrm{C}$, are shown in Figures 4 . The presence of oxidation layer (thickness $\sim 60 \mathrm{~nm}$ ) in the surface is clearly visible in the BF image. The SAED pattern of the surface layer is composed of three sets of diffraction patterns. The strong diffraction set outlined by the solid lines is attributed to metal Mo phase (BCC) with zone axis parallel to the [011] direction. Two weak diffraction sets can be indexed as monoclinic $\mathrm{MoO}_{2}$ 
phase with zone axis parallel to the [112] and [11-1] directions, respectively, as marked by the dash lines. Diffraction studies indicate that the surface area is a mixture of $\mathrm{Mo}$ and $\mathrm{MoO}_{2}$. HRTEM is used to identify the microstructure of the surface layer. HRTEM micrograph was taken from the surface with the electron beam oriented along [011] with respect to cubic Mo phase. It is interesting to note that the well-defined lattice fringe has a lattice spacing of 0.340 $\mathrm{nm}$, which is very close to that of the $(-111)$ lattice plane spacing $(0.341 \mathrm{~nm})$ of the monoclinic structured $\mathrm{MoO}_{2}$. HRTEM observation confirms the existence of $\mathrm{MoO}_{2}$ domains. Both electron diffraction and HRTEM results are in good agreement with GIXRD observations.

Using GIXRD, TEM and other characterization techniques, we observed that oxide formation of molybdenum in water vapor progresses from $\mathrm{Mo}$ to $\mathrm{MoO}_{3}$ and $\mathrm{MoO}_{2}$ at $600{ }^{\circ} \mathrm{C}$ and finally only $\mathrm{MoO}_{2}$ exists up to $1200{ }^{\circ} \mathrm{C}$. These oxidation processes accompany with the absolute weight loss increasing as the temperature increases. The results are generally agreement with the accepted mechanisms and knowledge regarding the oxidation of Mo. Oxidized molybdenum is susceptible to loss from the volatilization of $\mathrm{MoO}_{3}$ as both the oxide and the hydroxide $\mathrm{MoO}_{2}(\mathrm{OH})_{2}[17]$. The hydroxide is formed by the reaction [9]:

$\mathrm{MoO}_{3}(\mathrm{~s})+\mathrm{H}_{2} \mathrm{O}(\mathrm{g}) \rightarrow \mathrm{MoO}_{2}(\mathrm{OH})_{2}(\mathrm{~g})$

The loss of $\mathrm{MoO}_{3}$ agrees with the temperatures reported for the melting points of $\mathrm{MoO}_{3}\left(795{ }^{\circ} \mathrm{C}\right)$ and a $\mathrm{MoO}_{3}-\mathrm{MoO}_{2}$ eutectic $\left(778{ }^{\circ} \mathrm{C}\right)$ [9]. In the temperature range of $800-1200{ }^{\circ} \mathrm{C}$, the results obtained so far give evidence that under the given experimental conditions for the oxidation of Mo, $\mathrm{MoO}_{2}$ represents the only stable product phase.

One interesting TEM observation is that there is no $\mathrm{MoO}_{2}$ thin layer formed in the observed area at $600{ }^{\circ} \mathrm{C}$ and $800{ }^{\circ} \mathrm{C}$ samples, and $\mathrm{MoO}_{2}$ phase always coexists with Mo or both $\mathrm{Mo}$ and $\mathrm{MoO}_{3}$. 
This phenomenon is different from the previous reports $[4,9]$, in which a thin $\mathrm{MoO}_{2}$ layer was observed in the oxidized molybdenum in air. The result appears to suggest the complicated oxidation process of molybdenum, especially considering specific conditions including temperature, pressure, time, and medium (synthetic air vs. water vapor). Further work with advanced characterization techniques is needed to probe atomic and microstructural evolution, then determine the oxidation mechanism of molybdenum.

At the same temperature $\left(600{ }^{\circ} \mathrm{C}\right)$, molybdenum oxidation in air is so rapid and Mo is almost fully oxidized to $\mathrm{MoO}_{3}$. According to our experiments, under synthetic air environment, i.e. above $\sim 20 \mathrm{kPa}$ of oxygen partial pressure, $\mathrm{MoO}_{3}$ is the major oxide phase detected by XRD. As opposed to oxygen, when $\mathrm{H}_{2} \mathrm{O}$ is the oxidizing medium, the oxidation of Mo is significantly retarded which is confirmed by cross-sectional SEM observation, and Mo oxides $\left(\mathrm{MoO}_{2}\right.$ and $\mathrm{MoO}_{3}$ ) are minor phases in residual surface which is mixed with $\mathrm{Mo}, \mathrm{MoO}_{2}$, and $\mathrm{MoO}_{3}$. It should be noted that no intermediate molybdenum oxides were observed under both steam and air conditions in our study.

The present detailed analysis confirms that while the structural features that govern the oxidation of Mo in air remain generally unchanged when the oxidizing medium is instead water vapor, the effect of the significantly reduced partial pressure of oxygen evolved by water vapor at the temperatures investigated gives rise to some important differences. However, interpretation of the occurring reactions and the corresponding driving forces at present is merely speculative. Experiments are now in progress to explore the chemical and structural transformations of molybdenum under oxidizing and reducing atmospheres of relevance to LOCA analyzes in more detail. 


\section{Acknowledgement}

This work was supported by the U.S. Department of Energy, Office of Nuclear Energy, Fuel Cycle Research and Development program. 


\section{Reference}

[1] G. Schanz, B. Adroguer, and A. Volcheck, Nucl. Eng. Des. 232 (2004) 75-84.

[2] H. Chung, Nucl. Eng. Technol. 37 (4) (2005) 327-362.

[3] E. S. Jones, J. F. Mosher, R. Speiser, and J. W. Spretnak, Corrosion, 14 (1958) 20-26.

[4] E. Gulbrasen, K. Andrew, and F. Brassart, J. Electrochem. Soc. 110 (9) (1963) 952-959.

[5] R. Bartlett, J. Electrochem. Soc. 112 (7) (1965) 744-746.

[6] E. Gulbrasen, K. Andrew, and F. Brassart, J. Electrochem. Soc. 110 (7) (1963) 242-243.

[7] P. Kofstad, J. Inst. Of Metals, 90 (1962) 253-264.

[8] D. R. Olander and J. L. Schofill, Metallurgical Transactions, 1 (1970) 2775-2784.

[9] R. Speiser and G. R. St. Pierre, in The Science and Technology of Tungsten, Tantalum, Molybdenum, Niobium and Their Alloys ed. By N. E. Promisel, Pergamon Press (1964) 289330.

[10] G. Ramadorai, M. E. Wadsworth, and C. K. Hansen, Metallurgical Transactions, 6B (1975) 579-584.

[11] Bulletin of Alloy Phase Diagrams, 1-2 (1980) 85-89.

[12] A. T. Nelson, E. S. Sooby, Y-J. Kim, B. Cheng, S. A. Maloy, J. Nucl. Mater. 448 (2014) 441-447.

[13] B. Sudhirw and Rishi Raj, J. Am. Ceram. Soc., 89 [4] (2006)1380-1387. 
[14] A. Guinier, X-ray Diffraction in Crystals, Imperfect Crystals and Amorphous Bodies, (Dover, New York, 1994), p. 2.

[15] International Centre for Diffraction Data, Powder Diffraction File, Joint Committee on Powder Diffraction Standards, Philadelphia, PA, 1974-present.

[16] HSC Chemistry Software. http://www.hsc-chemistry.net/

[17] G. R. Smolik, D. A. Petti, S. T. Schuetz, Oxidation, Volatilization, and Redistribution of Molybdenum from TZM Alloy in Air, Idaho National Laboratory Technical Report, INEEL/EXT-99-01353, 2000. 


\section{Figure Captions}

Figure 1. (a) Evolution of GIXRD patterns during oxidation of Mo in synthetic air at temperatures of $500{ }^{\circ} \mathrm{C}$ and $600{ }^{\circ} \mathrm{C}$ (exposure time $\sim 240$ minutes). A diffraction pattern of pristine molybdenum metal is shown for comparison. Characteristic diffraction peaks of $\mathrm{MoO}_{3}$ and Mo metal are indicated. (b) Evolution of GIXRD patterns during oxidation of Mo in water vapor at temperatures of 600,800 , and $1200{ }^{\circ} \mathrm{C}$ (exposure times at $240,180,30$ minutes, respectively). A diffraction pattern of pristine molybdenum metal is shown for comparison. Characteristic diffraction peaks of $\mathrm{MoO}_{2}, \mathrm{MoO}_{3}$, and $\mathrm{Mo}$ metal are indicated.

Figure 2. (a) Cross-sectional SEM secondary electron image of molybdenum sample in synthetic air at a temperature of $600{ }^{\circ} \mathrm{C}$. (b) Cross-sectional SEM secondary electron image of molybdenum sample in water vapor at a temperature of $600{ }^{\circ} \mathrm{C}$.

Figure 3. (a) Cross-sectional TEM bright-field image (left) of molybdenum sample in water vapor at a temperature of $600{ }^{\circ} \mathrm{C}$ and SAED patterns (right) obtained from surface particles (labeled as $\mathrm{MoO}_{2}$ ), surface (labeled as $\mathrm{MoO}_{3}$ ), bulk (labeled as Mo), respectively. (b) High resolution TEM image obtained from the surface region of molybdenum sample in water vapor at a temperature of $600{ }^{\circ} \mathrm{C}$. Also shown as insets are magnified images from three different crystalline domains in the micrograph (denoted as $\mathrm{Mo}, \mathrm{MoO}_{2}$, and $\mathrm{MoO}_{3}$ ).

Figure 4. Cross-sectional TEM bright-field image (left) of molybdenum sample in water vapor at a temperature of $800{ }^{\circ} \mathrm{C}, \mathrm{SAED}$ patterns (top right) obtained from surface layer, and high resolution TEM image with $\mathrm{MoO}_{2}$ crystalline domain (bottom right). 


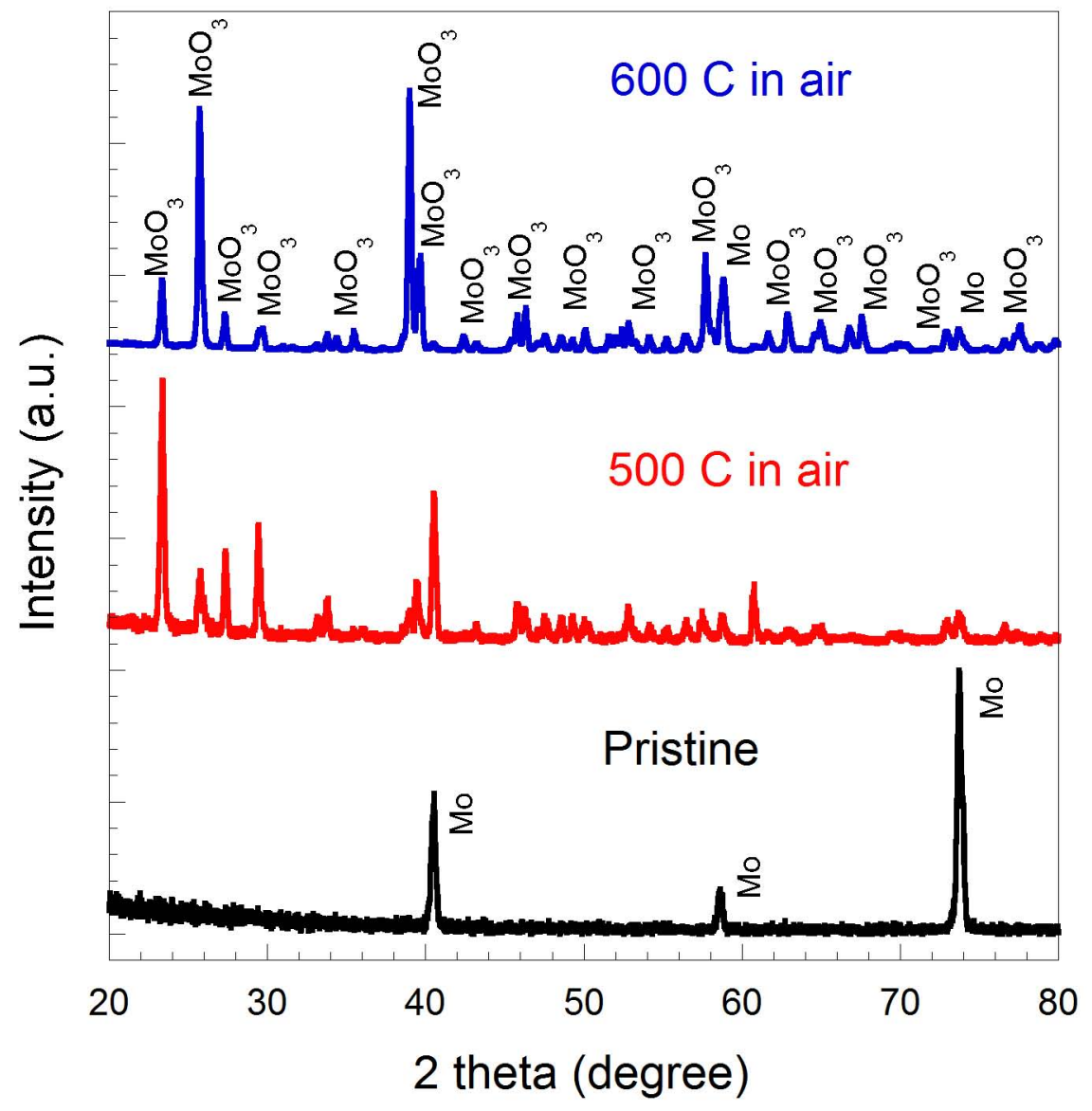


Figure $2 a$

\section{Oxidationlayer}

Mo substrate 
Mo substrate

Figure $2 b$ 
Figure 4

\section{$59.60 \mathrm{~nm}$}
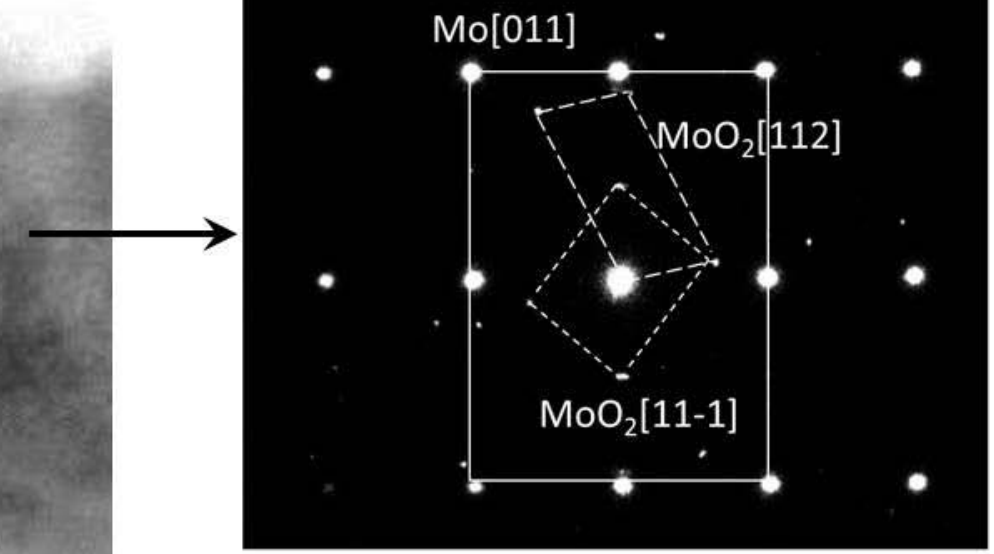

7.

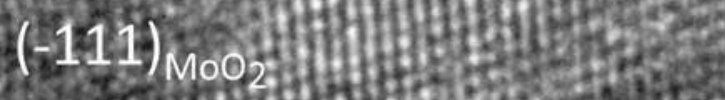

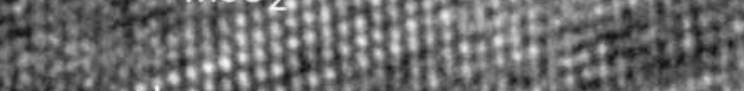

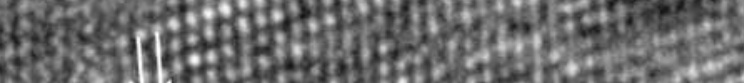

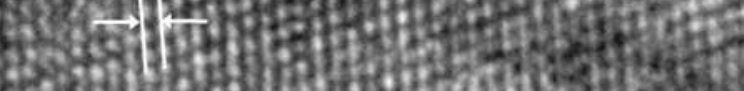
$530.3397 \mathrm{~nm}$ (1)

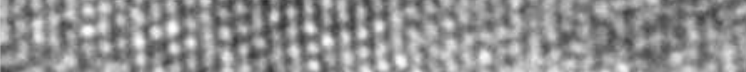

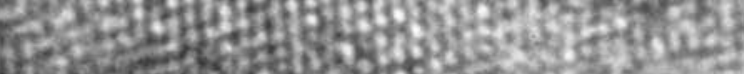
H.

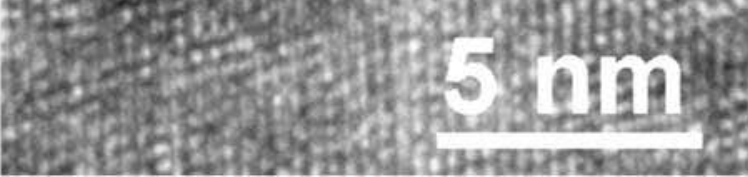


